\title{
Space-time measurements of oceanic sea states
}

\author{
Francesco Fedele ${ }^{\mathrm{a},{ }^{*}}$, Alvise Benetazzo ${ }^{\mathrm{b}}$, Guillermo Gallego ${ }^{\mathrm{c}}$, Ping-Chang Shih ${ }^{\mathrm{d}}$, \\ Anthony Yezzi ${ }^{\mathrm{d}}$, Francesco Barbariol ${ }^{\mathrm{e}}$, Fabrice Ardhuin ${ }^{\mathrm{f}}$ \\ ${ }^{a}$ School of Civil and Environmental Engineering, School of Electrical and Computer Engineering, Georgia Institute of Technology, Atlanta, GA, USA. \\ ${ }^{b}$ Institute of Marine Sciences, Italian National Research Council, Venice, Italy. \\ ${ }^{c}$ Grupo de Tratamiento de Imágenes, Universidad Politécnica de Madrid, Madrid, Spain. \\ ${ }^{d}$ School of Electrical and Computer Engineering, Georgia Institute of Technology, Atlanta, GA, USA. \\ ${ }^{e}$ Department of Civil, Environmental and Architectural Engineering, University of Padua, Padua, Italy. \\ ${ }^{f}$ Laboratoire d'Océanographie Spatiale, Ifremer, Brest, France.
}

\section{ARTICLE INFO}

\section{Article history}

Available online

\section{Keywords:}

\section{Stereo measurement}

WASS

Wave number-frequency spectrum

Wave statistics

Space-time extremes

\section{ABSTRACT}

\begin{abstract}
Stereo video techniques are effective for estimating the space-time wave dynamics over an area of the ocean. Indeed, a stereo camera view allows retrieval of both spatial and temporal data whose statistical content is richer than that of time series data retrieved from point wave probes. We present an application of the Wave Acquisition Stereo System (WASS) for the analysis of offshore video measurements of gravity waves in the Northern Adriatic Sea and near the southern seashore of the Crimean peninsula, in the Black Sea. We use classical epipolar techniques to reconstruct the sea surface from the stereo pairs sequentially in time, viz. a sequence of spatial snapshots. We also present a variational approach that exploits the entire data image set providing a global space-time imaging of the sea surface, viz. simultaneous reconstruction of several spatial snapshots of the surface in order to guarantee continuity of the sea surface both in space and time. Analysis of the WASS measurements show that the sea surface can be accurately estimated in space and time together, yielding associated directional spectra and wave statistics at a point in time that agrees well with probabilistic models. In particular, WASS stereo imaging is able to capture typical features of the wave surface, especially the crest-to-trough asymmetry due to second order nonlinearities, and the observed shape of large waves are fairly described by theoretical models based on the theory of quasideterminism (Boccotti, 2000). Further, we investigate the space-time extremes of the observed stationary sea states, viz. the largest surface wave heights expected over a given area during the sea state duration. The WASS analysis provides the first experimental proof that a space-time extreme is generally larger than that observed in time via point measurements, in agreement with the predictions based on stochastic theories for global maxima of Gaussian fields.
\end{abstract}

\section{Introduction}

The statistics and spectra of ocean waves are typically estimated from time series of the wave surface displacements retrieved from wave gauges a fixed point $P$ of the ocean. However, the limited information content of point measurements does not allow accurate predictions of the space-time wave dynamics over a given area around $P$ and the associated wave extremes. Only at large spatial scales, Synthetic Aperture Radar (SAR), or Interferometric SAR (INSAR) provides sufficient resolution for measuring waves longer than $100 \mathrm{~m}$ (see, e.g., Dankert et al., 2003). At smaller scales, video techniques are effective to correctly estimate the space-time dynamics and associated spectral properties (see, for example, Holland and Holman, 1997; Holland et al., 1997; Benetazzo, 2006). In particular, stereo cameras can be exploited to retrieve both spatial and temporal data whose statistical content is richer than that of a time series retrieved from wave gauges (Fedele et al., 2011b; Gallego et al., 2011; Benetazzo et al., 2012). In practice, stereopairs are acquired simultaneously and the geometry of the stereo system is defined so to minimize errors due to sea surface specularities (Jahne, 1993). Epipolar methods are traditionally used for the reconstruction of the water surface (Mat et al., 2004; see also Benetazzo, 2006), and recently variational-based techniques have been proposed to solve for the stereo problem (see for example, Gallego et al., 2011). In particular, epipolarbased techniques consist in finding pixel correspondences in the two synchronized images by a pixel-by-pixel-based search that is computationally expensive. Instead, variational-based approaches infer the waveform of the ocean surface as a minimizer of a composite energy functional that combines a photometric matching term along with regularization terms involving the smoothness of the unknowns. The desired ocean surface shape is the solution of a partial differential equation derived from the 
optimality conditions of the energy functional (Gallego et al., 2011).

Only in the last two decades or so, stereo imaging has become suitable for applications in oceanography. In particular, directional spectra of short gravity waves were estimated from stereo-pairs by Shemdin et al. (1988) and Banner et al. (1989). Benetazzo (2006) has proposed and tested a Wave Acquisition Stereo System (WASS) for field measurements at the coast, and more recently Bechle and $\mathrm{Wu}$ (2011) have studied coastal waves over areas $\sim 3 \mathrm{~m}^{2}$ using a trinocular system (Wanek and $\mathrm{Wu}$, 2006). Kosnik and Dulov (2011) have estimated sea roughness from stereo images and de Vries et al. (2011) have presented a stereo analysis of waves in the surf zone over an area of $1000 \mathrm{~m}^{2}$.

In this work, we present two applications of WASS for stereo measurements of gravity waves in the northern Adriatic Sea, off the Venice coast, and near the southern seashore of the Crimean peninsula, in the Black Sea. The reminder of this paper is organized as follows. We first provide an overview of the WASS deployments at the Italian and Crimean sites and then briefly discuss the image processing behind the stereo reconstruction of the wave surface using epipolar and variational techniques. In particular, we present a variational approach that exploits the entire space-time data image set providing a simultaneous reconstruction of a time-ordered stack of spatial snapshots of the surface in order to guarantee continuity of the sea surface both in space and time. Then, we evaluate directional spectra and the time wave statistics at a given point is compared against theoretical models. Finally, we investigate the properties of space-time extremes of the observed sea states, viz. the largest surface wave heights expected over a given area during the sea state duration.

\section{2. WASS Deployments and data acquisition}

In June 2009 WASS was deployed at the oceanographic tower Acqua Alta (Cavaleri et al., 1997) located in the Northern Adriatic Sea, 10 miles off the coastline of Venice, in 16 meters deep waters. Video measurements were acquired in three experiments carried out during the period 2009-2010 to investigate both space-time and spectral properties of oceanic waves (Fedele et al., 2011a,b; Benetazzo et al., 2012). To maximize the common field of view of the two cameras, i.e. stereo pairs, WASS was deployed at approximately $12.5 \mathrm{~m}$ above the mean sea level on the third floor of the platform (see Fig. 1, left). In such a setting, the stereo pairs cover a common trapezoidal area of approximately $1100 \mathrm{~m}^{2}$, with sides of length $30 \mathrm{~m}$ and $100 \mathrm{~m}$, respectively, and a width of $100 \mathrm{~m}$. The deployed WASS system includes two BM-500GE JAI digital cameras $(2456 \times 2058 \mathrm{CCD}$ active pixels with a square cell size of $3.45 \mu \mathrm{m} \times 3.45 \mu \mathrm{m}$, and a 8-bit based dynamical range) and an acquisition workstation (Figure 1, center). The cameras are set at $2.5 \mathrm{~m}$ apart and they mount $5-\mathrm{mm}$ focal length low distortion lenses (21-mm equivalent focal length in a $35-\mathrm{mm}$ film camera). Camera synchronization is attained by an external trigger that allows different frame acquisition rates $(5,10$, and $15 \mathrm{~Hz})$ and the delay is kept within $1 \mathrm{~ms}$ as required for wave measurements (Holthuijsen, 1983). A dedicated Giga Ethernet device and high performance Hard Drives are used to manage digital image data transfer rate up to $75 \mathrm{Megabyte} / \mathrm{s}$. The calibration of the WASS system is performed by means of standard image analysis techniques (Ma et al., 2004). These exploit a given known reference (for example, a chess-board) to estimate internal parameters such as lens focal length, principal point and distortion, i.e., lens angular aberration as well as external parameters, which yield the reciprocal position of the two cameras with respect to a fixed reference world system. In this work we will elaborate the stereo data acquired during Experiments 2 and 3, viz. 21000 and 9000 snapshots, respectively. Both experiments were conducted during NorthEastern wind (namely Bora) conditions with mean wind speed and significant wave height equal to $(9.6 \mathrm{~m} / \mathrm{s}, 1.09 \mathrm{~m})$ and $(17.9$ $\mathrm{m} / \mathrm{s}, 2.16 \mathrm{~m}$ ) for Experiments 2 and 3, respectively (see Benetazzo et al. 2012).

In October 2009, another WASS was also mounted 12 meters above the mean sea level on the research platform of the Marine Hydrophysical Institute of Ukraine, located in Katsiveli, at the southern tip of the Crimean peninsula, between Sevastopol and Yalta (see Fig. 1, right). At the platform the water depth is approximately $30 \mathrm{~m}$. For this experiment we deployed a couple of JAI CM-200 GE digital cameras $(1624 \times 1236$ CCD active pixels with a square cell size of $4.40 \mu \mathrm{m} \times 4.40 \mu \mathrm{m}$, and an 8-bit based dynamical range), set $2.5 \mathrm{~m}$ apart and with 5 -mm lenses (25-mm equivalent focal length). The system was oriented to the east-south-east, with moderate sun reflections in the morning, and was operated at an acquisition frequency of $10 \mathrm{~Hz}$ producing 21000 pairs of images. We analyzed 6000 pairs of a sea state with significant wave height $\sim 0.26 \mathrm{~m}$ and wind speed $\sim 7.5 \mathrm{~m} / \mathrm{s}$. The internal and external camera parameters and geometry of the stereo pair were calibrated using the standard procedures mentioned above. The resulting imaged and processed areas were approximately $50 \times 50 \mathrm{~m}^{2}$ and $15 \times 15 \mathrm{~m}^{2}$, respectively. The pixel footprint of the imaged area gives an estimate of WASS resolution on the horizontal plane, which is approximately 40 $\mathrm{mm}$ and $20 \mathrm{~mm}$ for Acqua Alta and Crimean experiments, respectively. In the epipolar method, the use of a sub-pixel detection algorithm did improve the resolution by a factor 5-10 (see Benetazzo et al. 2012, Nobach, H., Honkanen, M., 2005). As regard to vertical resolution, the WASS system is intrinsically a high-resolution system, because the Z-coordinate is a continuous function of both left and right image coordinates as well as internal/external parameters. On the other hand, the accuracy in measuring the wave surface elevation is defined for a given WASS configuration (see Benetazzo, 2006; Benetazzo et al., 2012), and it was estimated equal to $0.01 \mathrm{~m}$ and $0.04 \mathrm{~m}$ for
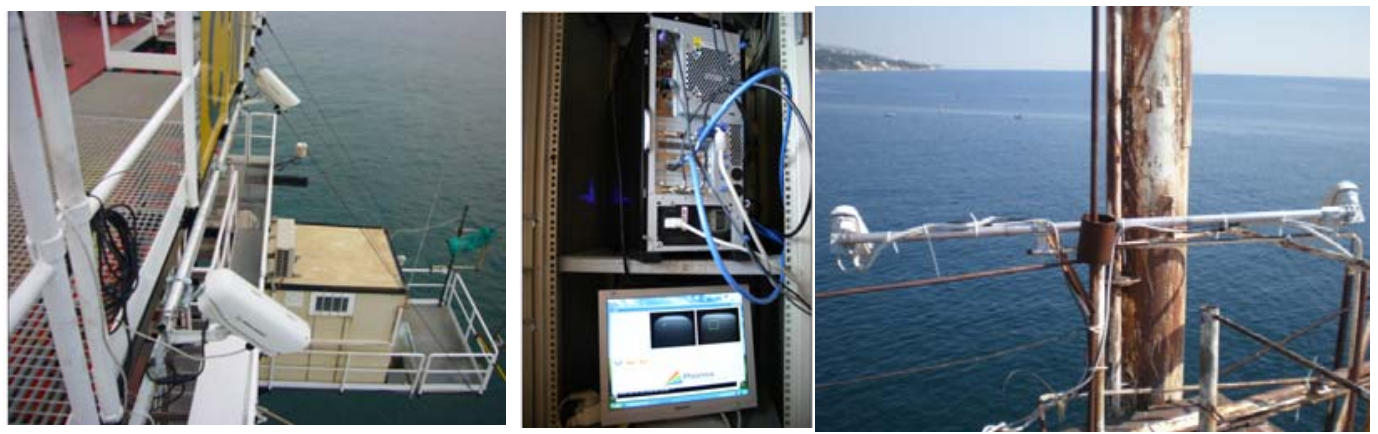

Fig. 1. (Left) deployed WASS at Acqua Alta, (center) workstation and cables, and (right) deployed WASS at the Crimean site. 
Acqua Alta and Crimean experiments respectively. At the present stage of WASS development, specular reflections restricted the application of stereo methods to conditions with limited sun glitter. The acquired stereo sequences satisfy this condition. A solution accounting for the sun position with respect to the stereo system could alleviate this drawback, but it is beyond the goal of this study. To remove unwanted spikes (outlier) from disparities we used the algorithm described in Goring and Nikora (2002). We found that WASS estimates of wave parameters fairly agree with those from standard wave gauge instruments available at Acqua Alta (see Benetazzo et al. 2012).

Acqua Alta stereo data were processed using epipolar methods and the reconstructed wave surface displacements were validated against gauge measurements available at the tower (Benetazzo et al., 2012). Crimean data were processed using a technique based on variational methods, combining the stereo disparity method in (Alvarez et al., 2002) with the enforcement of temporal coherence in (Gallego 2011, Ch.7), as discussed in the next section below. Studies are in progress to compare the performance of epipolar against variational methods for both data sets and this will be discussed elsewhere.

\section{Stereo processing}

Given stereo images in favorable acquisition conditions, it is possible to recover the three-dimensional (3-D) structure of the scene (shape of the ocean surface) using computer vision techniques. Following the classical image-based (bottom-up) approach, the 3-D reconstruction problem is split in two: the establishment of point correspondences across images (stereomatching) and depth recovery (triangulation or back-projection of point matches). The first sub-problem is more difficult than the second. Epipolar geometry is exploited to guide the search of point matches, i.e., the establishment of a disparity map between images (see, for example, Ma et al., 2004).

In traditional methods, correspondences are established by maximization of some cross-correlation photometric score along epipolar lines. This enforces at most one-dimensional (1-D) continuity of the disparity map (along the epipolar lines). The dense disparity method (Alvarez et al., 2002) focuses on solving the stereo matching problem for surfaces that can be represented as depth maps from one of the cameras by minimization of a cost functional in a variational framework. This enforces continuity of the solution (disparity map) in the full two-dimensional (2-D) image domain. Variational methods provide a solid mathematical framework that improves the robustness of the reconstruction against image noise and other error sources (Gallego et al., 2011). Inspired by the dense disparity method, we develop a cost functional with a data fidelity term (which measures the photometric mismatch between transferred image intensities according to a candidate disparity function) and a smoothing term (regularizer) in the only unknown of the problem: the tangential disparity map. We further extend this method to enforce continuity of the solution not only in space but also in time (Gallego, 2011, Ch. 7), considering a cost functional whose data fidelity term is the sum of the snapshot-wise data fidelity terms and whose regularizer comprises penalties on the temporal derivatives of the tangential disparity:

$E_{\text {data }}(\lambda)=\frac{1}{T} \int_{T} \int_{\Omega}^{1} \frac{1}{2}\left(I_{1}\left(x_{1}, t\right)-I_{2}\left(x_{2}, t\right)\right)^{2} d x_{1} d t \#$
$E_{\text {smooth }}(\lambda)=\frac{1}{T} \int_{T} \int_{\Omega} \frac{1}{2}\|\nabla \lambda\|^{2} d x_{1} d t$

Here, $I_{1}$ and $I_{2}$ are the acquired video data by both cameras, $\lambda\left(x_{1}, t\right)$ is the tangential disparity map for all snapshots and $\nabla \lambda$ is the spatio-temporal gradient of the tangential disparity. Coordinates $\mathrm{x}_{1}$ and $\mathrm{x}_{2}$ are 2-D vectors that refer to corresponding locations in images 1 and 2, respectively. Point $x_{2}$ is obtained from $x_{1}$ via formula $x_{2}=x_{1}+d(\lambda)$, where $d(\lambda)$ is the disparity. We also incorporate a parameter that controls the relative amount of temporal coherence with respect to the spatial one. The necessary optimality condition of the above cost functional is given by the Euler-Lagrange (EL) equation

$\alpha \Delta \lambda+\left(I_{1}\left(x_{1}, t\right)-I_{2}\left(x_{2}, t\right)\right)\left(\partial I_{2}\left(x_{2}, t\right) / \partial \lambda\right)=0 \#$

and homogeneous Neumann boundary conditions, where the above Laplacian $\Delta \lambda$ comprises second order derivatives in spacetime. The partial differential equation (PDE) is solved via the steady state of a gradient descent method in the unknown function. The PDE is discretized using finite differences and it is numerically solved via 3-D multigrid methods (Briggs et al., 2000; Trottenberg, 2000), which are the standard numerical tools to solve non-linear elliptic boundary value problems such as the one considered here. Hence, this method consists of simultaneous estimation of the disparity maps for all snapshots in a sequence (see Fig. 2). Note that this is not an extension of the variational approach of Gallego et al. (2011), which directly computes the surface of the ocean without using a disparity map.

The proposed method was applied to a sub-sampled version of the images (by a factor of 4 in both spatial dimensions) to be able to process a significant group of snapshots simultaneously using a dual-core desktop computer (4097 snapshots). A disparity map with 2572 points was chosen (Fig. 2 shows a portion of 1025 snapshots of the reconstructed disparity). Given the disparity maps, it is straightforward to compute the depths of the corresponding 3-D points and their heights with respect to an average plane via triangulation (see Hartley and Sturm, 1997).

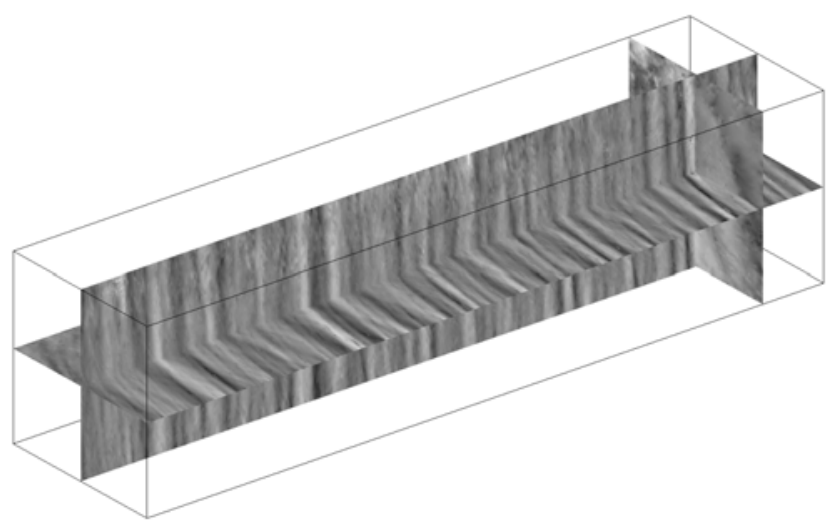

Fig. 2. Crimean experiment. Slices of the space-time tangential disparity. Grayscale encoded, from dark (low) to white (high). The mean disparity over all snapshots has been removed to better visualize the oscillating patterns in the disparity due to the wave displacement caused by wind waves. Long axis is time $t$. The grid, of size $257 \times 257 \times 1025$ points, in the image-time domain corresponds to a physical space-time volume of size 20 $\times 15 \mathrm{~m}^{2}$ by $102.5 \mathrm{~s}$ 

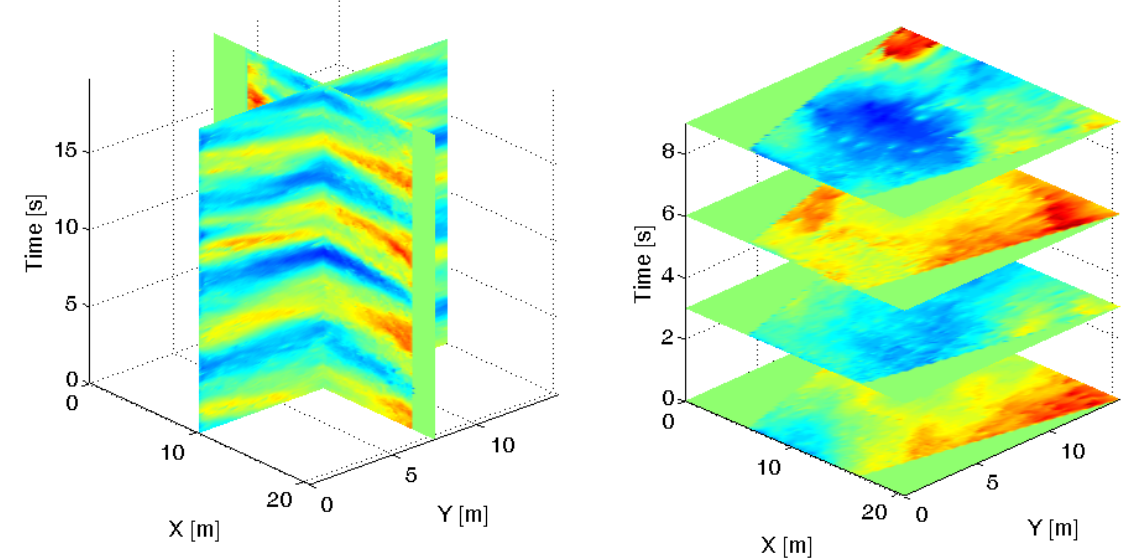

Fig. 3. Crimean experiment: horizontal (right) and vertical (left) slicing of the space-time surface height volume $V$. Spatial resolution: $10 \mathrm{~cm}$. Covered area: $20 \times 15 \mathrm{~m}^{2}$. Processed area (inside the trapezoid): $15^{2} \mathrm{~m}^{2}$.

In simple words, both epipolar and variational methods yield the stereo reconstruction of the wave surface as a four dimensional (4-D) manifold $Z=\eta(X, Y, t)$ defined on a 3-D spacetime volume $V$ with sides $X_{\max }=35 \mathrm{~m}, Y_{\max }=35 \mathrm{~m}$ and $t_{\max } \sim 30$ min along $X, Y$ and $t$, respectively. As an example, horizontal and vertical projections of the wave surface manifold are shown in Fig. 3 from the elaboration of Crimean data.

In the following, we will estimate directional spectra from the reconstructed wave surface and the observed wave extremes in space-time will be compared against theoretical models based on Euler Characteristics (Adler, 1981; Adler, 2000; Adler and Taylor, 2007).

\section{Directional spectra}

Video data were collected by WASS during three field campaigns at Acqua Alta in 2009-2010. The three experiments cover a broad range of wave height conditions as discussed in Fedele et al. (2011) and Benetazzo et al. (2012). WASS estimates were compared against reference point time series measurements provided by wave gauge instruments available at Acqua Alta. Such comparison was done by first selecting a virtual point probe within the reconstructed area and then extracting the associated time series of the stereo reconstructed wave surface displacements. Given the wave time series, various statistical and spectral properties of waves were computed and compared against those from Acqua Alta instrument. We refer to Fedele et al. (2011b) and Benetazzo et al. (2012) for such comparisons, which provide evidence that the accuracy of WASS measurements is comparable to that of more traditional wave instruments.

From the stereo data acquired in Experiment 2 at Acqua Alta (21000 snapshots at $10 \mathrm{~Hz}$ over an area of $\sim 1100 \mathrm{~m}^{2}$ ) the directional spectrum is estimated using a modified version of the MATLAB ${ }^{\circledR}$ DIWASP v1.3 toolbox (Johnson, 2004) for the Extended Maximum Entropy Principle (EMEP, see Hashimoto et al, 1994). Note that this technique estimates only a few directional Fourier coefficients of the directional spreading function from time series extracted at a limited number of virtual probes. Thus, for multimodal or complex directional spectra of the sea state EMEP has some limitations and the characterization of the directional spreading may be affected by noise. For Acqua Alta, the polar representation of the estimated $S(k, \theta)$ via EMEP is reported in Fig. 4.

The analysis of Crimean data is done using the variational disparity approach presented in section 3 , which provides the simultaneous reconstruction of the wave surface both in space and time. The estimate of the frequency spectrum $S(f)$ is reported in Fig. 5 (left), which shows an inertial range as $f^{4}$ in agreement with weak wave turbulence (Zakharov, 1999). Above $1 \mathrm{~Hz}$ the increased spectral slope is due to noise and the $f^{5}$ Phillips' regime is not observed (Phillips, 1958; Phillips, 1977; Newell and Zakharov, 2008). Clearly, the sea state includes both a swell and wind wave components with peak periods at $6 \mathrm{~s}$ and $3 \mathrm{~s}$, respectively. In the right panel of the same Figure we report a $k_{x}$ slice of the wavenumber-frequency spectrum $S\left(k_{x}, k_{y}, f\right), \quad k_{x}$ and $k_{y}$ being the wavenumbers along $X$ and $Y$ respectively. Note that the observed dispersion pattern deviates from the theoretical dispersion curve (dash line) with no current, viz. $k_{y}=(2 \pi f)^{2} / \mathrm{g}$ due to the Doppler-shift caused by underwater currents. Their speed is estimated as $\sim 0.2 \mathrm{~m} / \mathrm{s}$ by matching the theoretical wave-current dispersion curve (solid line) with the observed trend, and we refer to Fedele et al. (2011b) and Benetazzo et al. (2012) for a more detailed description of the estimation method. We also observe the signature of second-order harmonics due to the nonlinearity of the wave field, viz. the weak green line structure over the dispersion relation branch with positive Doppler shift in frequency.

The directional spectrum estimated using the EMEP method is shown in Fig. 6. In agreement with the frequency spectrum of

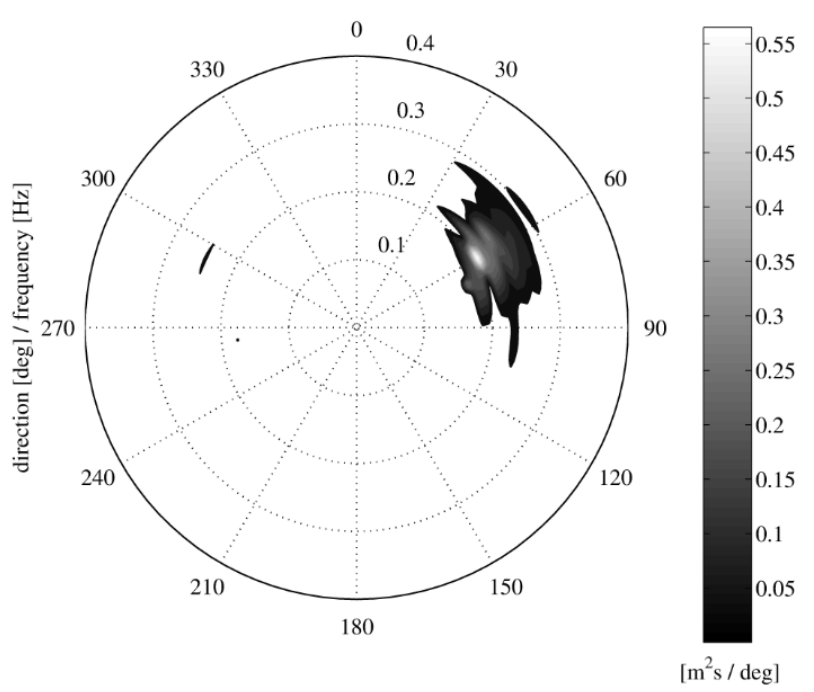

Fig. 4. Acqua Alta, Experiment 2: directional spectrum $S(k, \theta)$ estimated using EMEP method. Wave direction is measured clockwise from the geographic North (wind speed $9.6 \mathrm{~m} / \mathrm{s}$ and direction $51.2^{\circ} \mathrm{N}$ ). Resolution is $2^{\circ}$ and $10^{-2} \mathrm{~Hz}$. 

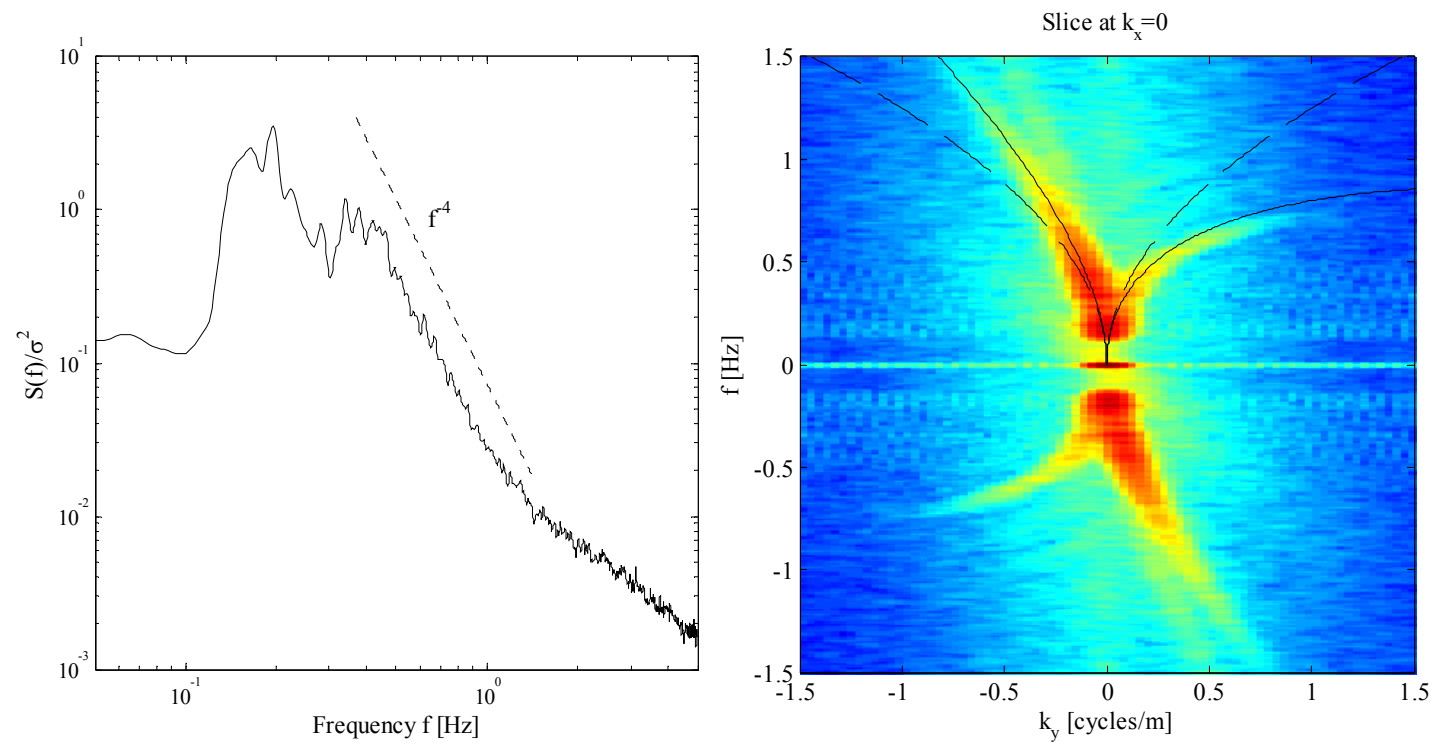

Fig. 5. Crimean experiment. Left: Observed mean frequency spectrum $S(f)$ estimated from time series extracted at 25 virtual probes within the imaged area. Nyquist frequency is $5.0 \mathrm{~Hz}$. Right: Slice of the 3-D spectrum $S\left(k_{x}, k_{y}, f\right)$ of wave heights at $k_{x}=0$. The theoretical wave dispersion curves with and without current are also shown (dash and solid lines respectively, current speed=0.2 m/s). Nyquist frequencies: 10 cycles $/ \mathrm{m}$ (space) and $5 \mathrm{~Hz}$ (time). Spectral resolutions: 0.0195 cycles/m (space) and $0.02 \mathrm{~Hz}$ (time).

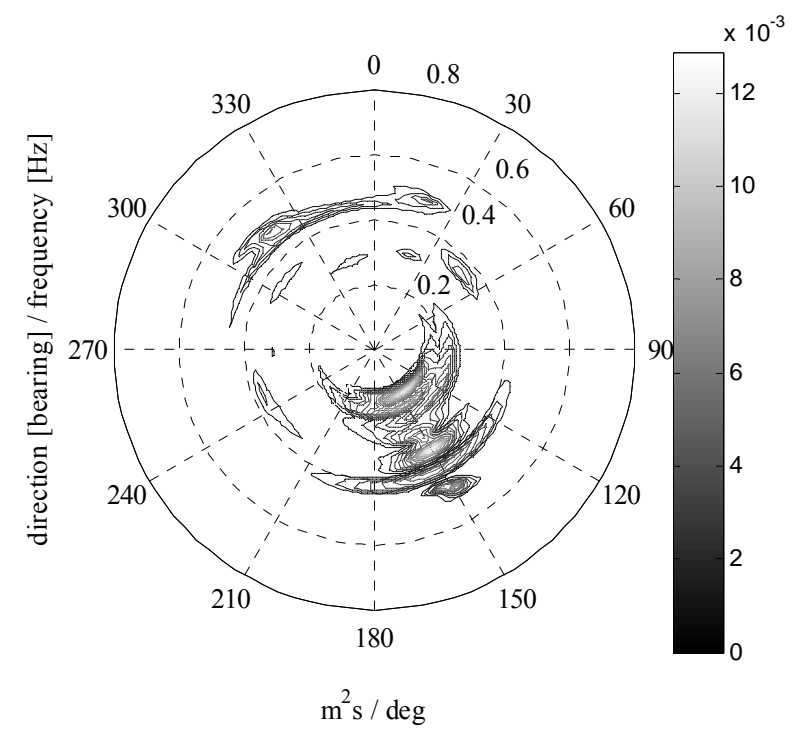

Fig. 6. Crimean experiment. Crimea experiment. Directional spectrum $S(k, \theta)$ estimated using EMEP method. Wave direction is measured clockwise from the geographic North (wind speed $7.5 \mathrm{~m} / \mathrm{s}$ and direction $150^{\circ} \mathrm{N}$ ). Angular (direction) and radial (frequency) resolutions are $5^{\circ}$ and $2 \times 10^{-2} \mathrm{~Hz}$, respectively.

Fig. 5 we observe both swell and wind wave components as incident waves toward the platform as well as some noise due to the abovementioned limitations of the EMEP estimator. Note also a reflected wave component due possibly to diffraction effects of the tower.

\section{Time Extreme Value Analysis}

In this Section we elaborate on the statistics of the largest waves expected at a given point of the imaged area in time, hereafter referred to as time extremes. We consider the stereoimaged wave surface acquired from Experiment 2 at Acqua Alta data, and we refer to Benetazzo et al. (2012) for the analysis of the video data acquired in Experiments 1 and 3. Consider the ensemble of the time series of the stereo reconstructed wave displacements extracted at several virtual probes. The time series are filtered above $1.1 \mathrm{~Hz}$ to remove noise, with variations of the wave variance less than $0.5 \%$. The expected maximum wave height $Z(N)=<H \quad / \sigma>$ of $N$ observed waves could then be easily estimated from the wave ensemble. According to Gumbel (1958), its theoretical value is given by (see also Tayfun and Fedele, 2007)

$$
Z(N)=\sqrt{\log \left(c_{0} N\right) / c_{1}}+\frac{\gamma_{e}}{2 \sqrt{c_{1} \log \left(c_{0} N\right)}}, \#
$$

where $\gamma_{e} \approx 0.5772$. For the Rayleigh (R) model the coefficients $\mathrm{c}_{0}=1, \mathrm{c}_{1}=1 / 8$, and for the Boccotti (B) model (Boccotti, 2000)

$c_{0}=(1+b) / \sqrt{2 b(1-a)}, c_{1}=0.25 /(1-a) \#$

with $a=-\psi^{*}, b=1$. Here, the parameter $\psi^{*}$ depends upon the first minimum of the wave covariance and $\sigma$ is the standard deviation of the wave surface. In particular, the average value over the time series ensemble is $\psi^{*} \approx 0.66$. Data are compared against $\mathrm{R}$ and $\mathrm{B}$ in Fig. 7. As one can see, $R$ overestimates the observed expected maximum wave heights as expected, because it does not account 


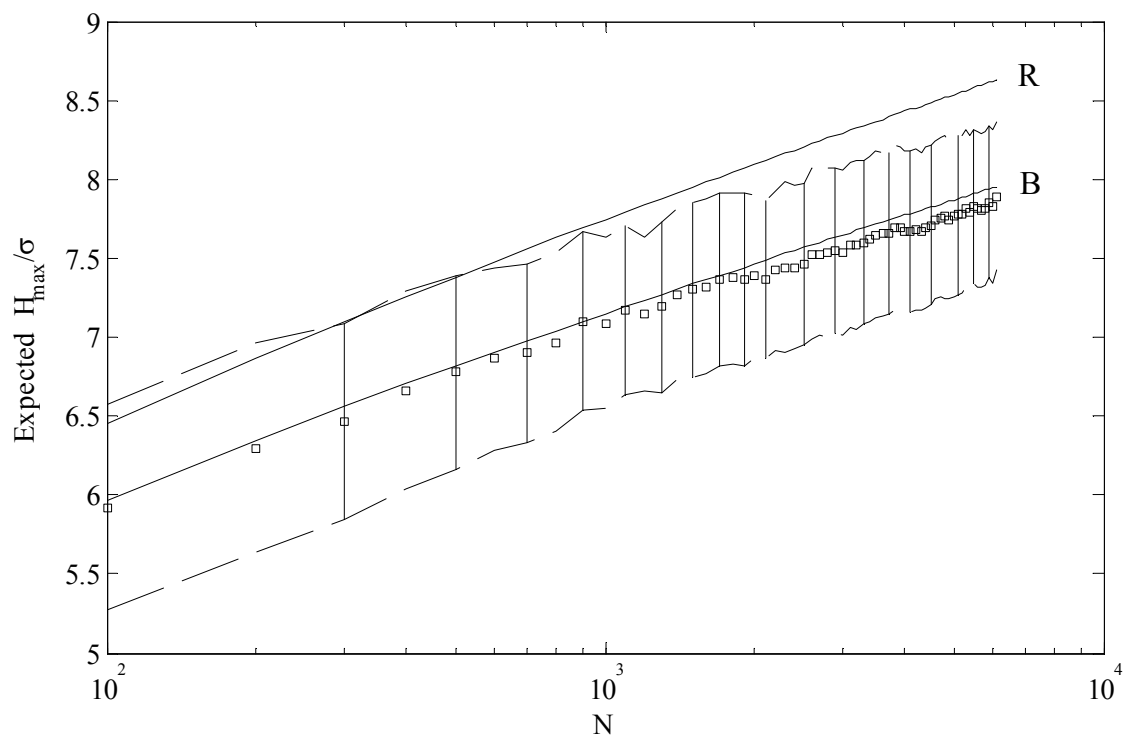

Fig. 7. Acqua Alta, Experiment 2: Expected maximum crest height: observed average max wave height amplitudes (square dots) and relative stability bands $Z(N) \pm \sigma_{N}, \sigma_{N}$ being the associated standard deviation, and theoretical $\mathrm{R}=$ Rayleigh, $\mathrm{B}=$ Boccotti models, respectively.

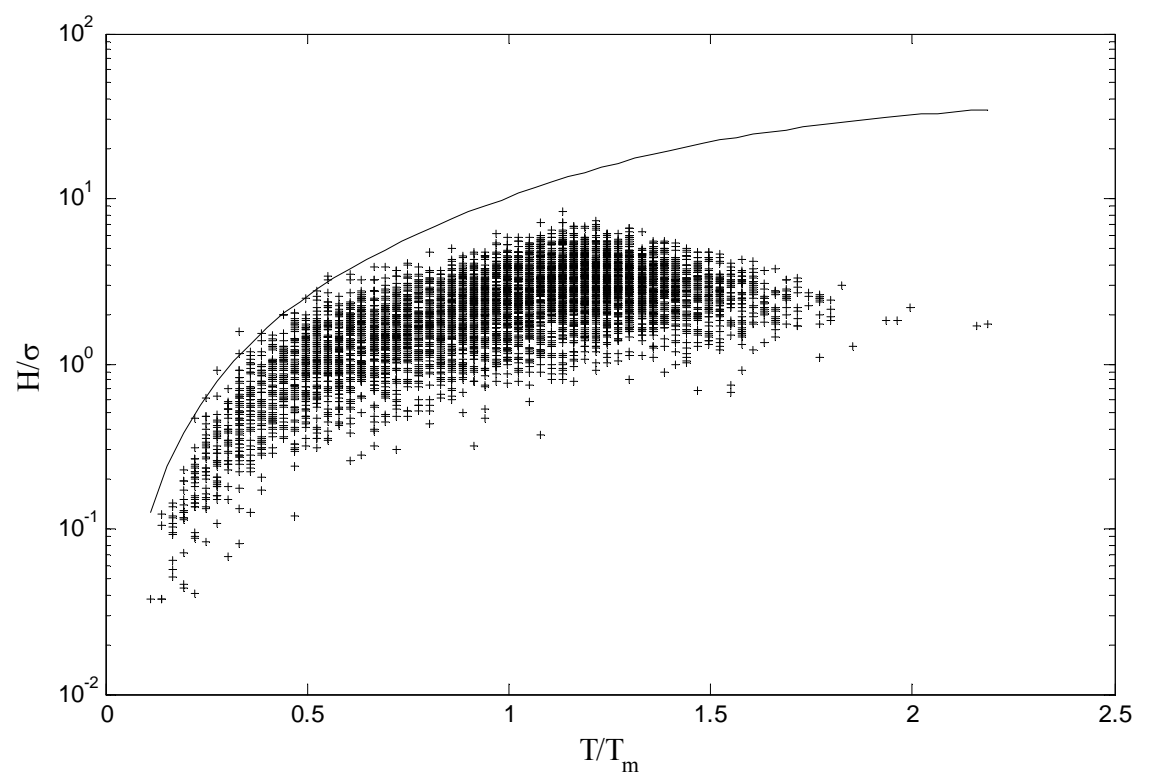

Fig. 8. Acqua Alta, Experiment 2: Theoretical Miche-Stokes upper bound (black line) for the crest-to-trough height $H$ of a wave with period $T$ and observed pairs $\left(T / T_{m}, H / \sigma\right)$ (markers), with $\sigma$ and $T_{m}$ as the wave surface standard deviation and mean wave period, respectively.

for bandwidth effects as B does (see also Boccotti, 2000; Benetazzo et al., 2012). Finally, we observe that the StokesMiche upper bound (Michell, 1893)

$\frac{H}{\sigma} \leq \frac{2 \pi}{7} \frac{\tanh (k d)}{k \sigma} \#$

is not violated by the largest waves observed at the different virtual probes as shown in Fig. 8. Here, $\mathrm{H}$ is the crest-to-trough height of a wave with wavenumber $k=2 \pi / L_{m}, L_{m}$ being the wavelength associated to the mean wave period $T_{m}$ via the linear dispersion relation.

\section{Expected shape of large waves}

Herafter we will compare the observed waveform of large waves against their expected theoretical shape (Lindgren, 1970; Boccotti, 2000). Consider among the observed waves, those with large crest-to-trough amplitudes $\alpha=H / \sigma>>1$. For dominant second order nonlinearities, the shape of these large waves tends, for $\alpha>>1$, to the deterministic form (Fedele, 2005; Fedele, 2008; Tayfun and Fedele, 2009)

$$
\begin{gathered}
<\eta(t) \mid \alpha>=\frac{\alpha}{2} \frac{\psi(t)-\psi\left(t-T^{*}\right)}{1-\psi\left(T^{*}\right)}+\frac{\mu \alpha^{2}}{8} \times \\
\left\{\left[\frac{\psi(t)-\psi\left(t-T^{*}\right)}{1-\psi\left(T^{*}\right)}\right]^{2}-\left[\frac{\hat{\psi}(t)-\hat{\psi}\left(t-T^{*}\right)}{1-\psi\left(T^{*}\right)}\right]^{2}\right\},
\end{gathered}
$$

where $\psi(t)$ is the normalized wave covariance, $\hat{\psi}(t)$ its Hilbert transform, and $T^{*}$ is the abscissa of the first absolute minimum of $\psi$ (Boccotti, 2000). The wave steepness can be estimated directly from data via time averages as $\mu=\left\langle\eta^{3}\right\rangle$, where $<\bullet>$ denotes expectation. A statistically stable estimate for $\mu$ can be defined from the moments of the wave spectrum as (Fedele, 2009; Fedele and Tayfun, 2009)

$\mu_{a}=\mu_{m}\left(1-v+v^{2}\right) \#$ 
where $v$ is the spectral bandwidth given by $v=\sqrt{m_{0} m_{2} / m_{1}^{2}-1}$, where $m_{j}$ are the spectral moments and $\mu_{m}$ is the steepness for narrowband waves, viz. $\mu_{m}=\sigma\left(\omega_{m}\right)^{2} / g$ with $\omega_{m}=m_{1} / m_{0}$ as the mean up-crossing frequency (Tayfun, 1986). The observed values for these parameters for Exp. 2 at Acqua Alta are given by $\mu=0.08, \mu_{m}=0.09, \mu_{a}=0.069$, and $v=0.52$ respectively. Further, if we define $C$ and $T$ as the crest and trough amplitudes of the same wave, given $\alpha>>1$, the conditional ratio $C / T$ is given by

$\{C / T \mid H=\alpha\}=\frac{1+2 \gamma Z}{1-2 \gamma Z} \cdot \frac{1+\mu \alpha(1+2 \gamma Z) / 4}{1-\mu \alpha(1-2 \gamma Z) / 4}, \#$

where $\mathrm{Z}$ is Gaussian with mean zero and unit variance and

$\gamma=\frac{1}{\alpha} \sqrt{\frac{1+\psi\left(T^{*}\right)}{2}}$ \#

Fig. 9 shows a fair agreement between the observed shape of large waves and the theoretical expected form (7) $\left(\mu=\mu_{m}=0.09\right.$ and $\mu_{a}=0.069$ and $\left.\psi\left(T^{*}\right)=-0.66\right)$. Further, Fig. 10 shows that the observed conditional ratio agrees well with the associated theoretical expected value from (9). Consider now among the observed waves, those with large crest amplitudes $\xi=h / \sigma>>1$. Their shape tends to

$<\eta(t) \mid \eta(0)=\xi>=\xi_{1} \psi(t)+\frac{\mu \xi_{1}^{2}}{2}\left[\psi^{2}(t)-\hat{\psi}^{2}(t)\right], \#$

where $\xi=\xi_{l}+\mu\left(\xi_{l}\right)^{2} / 2$ (Tayfun and Fedele, 2007; Fedele, 2008, 2009; Fedele and Tayfun, 2009). Fig. 11 shows a comparison between the observed shape of the wave with the largest crest and the respective theoretical expected wave profile from (11). Clearly, WASS stereo imaging is able to capture typical features of the wave surface, especially the crest-to-trough asymmetry due to second order nonlinearities, and observations are in fair agreement with theoretical models.

\section{Space-time extreme value analysis}

Recently some authors (Krogstad et al., 2004; Forristall, 2006; and non-Gaussian seas to show that the maximum surface height over a given area of the ocean is generally larger than that observed in time via point measurements. Indeed, in short-crested seas the surface time series gathered at the given location tends to underestimate the true actual wave surface maximum that can occur over a given region of area $A$ around $P$. The probability that the group passes at its apex through $P$ is practically null, because large waves travel on top of wave groups. The large crest height recorded in time at $P$ is simply due to the dynamical effects of a wave group that focuses nearby that location within or outside $A$ forming a larger wave crest. Only in narrow-band sea states, point measurements are exact in predicting such maximum, which is expected to be the same at any point in space. However, realistic oceanic conditions are generally shortcrested and extremes over an area are larger than those measured at a fixed point because point-probe measurements are not able to detect true maxima of short crested seas. As pointed out by Baxevani and Richlik (2004), the occurrence of an extreme in a Gaussian field is analogous to that of a big wave that a surfer searches for and always finds, because he moves around a large area instead of waiting at a fixed location for the big wave to occur. Indeed, if he spans a large area the chances to encounter the largest crest of a wave group increase, in agreement with the findings of the recent European Union 'MaxWave' project (Rosenthal and Lehner, 2008).

The prediction of spatial extremes is relevant in offshore engineering: the design of the air gap under the deck of fixed offshore structures should be based on the largest wave surface height over the rig's area footprint. The underestimation of the design height may be the cause of localized damages sometimes observed on the lower decks of platforms after storms (Forristall, 2006; Forristall, 2007).

In the following, we will show that WASS measurements allow for a complete statistical analysis of wave extremes exploiting Adler's Euler Characteristics (Adler, 1981; see also Piterbarg, 1995). Drawing from Fedele et al. (2011a,b) and Fedele (2012) we first consider wave surface maxima that occur over a given area at a fixed instant of time (space extremes) and then extend the analysis to global maxima over an area during the sea state duration (space-time extremes).

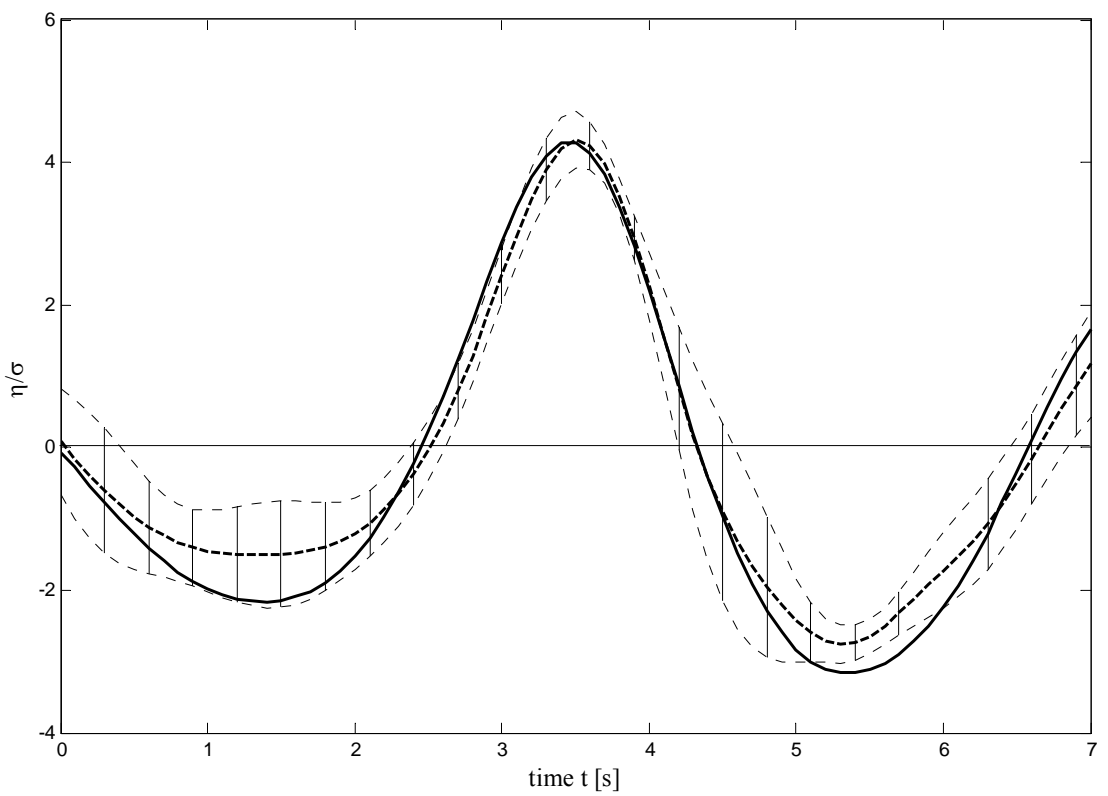

Fig. 9. Acqua Alta, Experiment 2: Observed shape of large waves (dashed line) given the crest-to-trough height $H$ in $[7 \sigma, 8 \sigma]$ and respective stability bands, and theoretical expected wave profile of Eq. (7) (solid line).

Forristall, 2007) carried out numerical simulations of Gaussian 


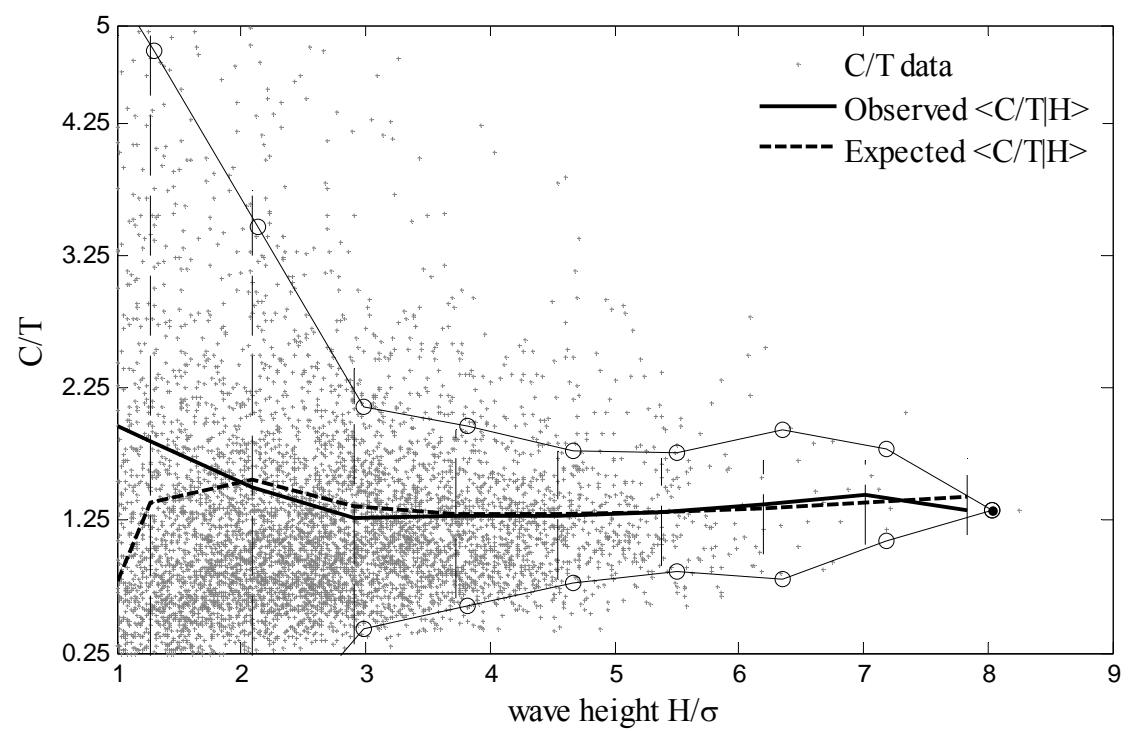

Fig. 10. Acqua Alta, Experiment 2: Observed conditional averages of $C / T$ (solid line) given the crest-to-trough height $H$ and the associated theoretical expected values from Eq. (9) (dashed line).

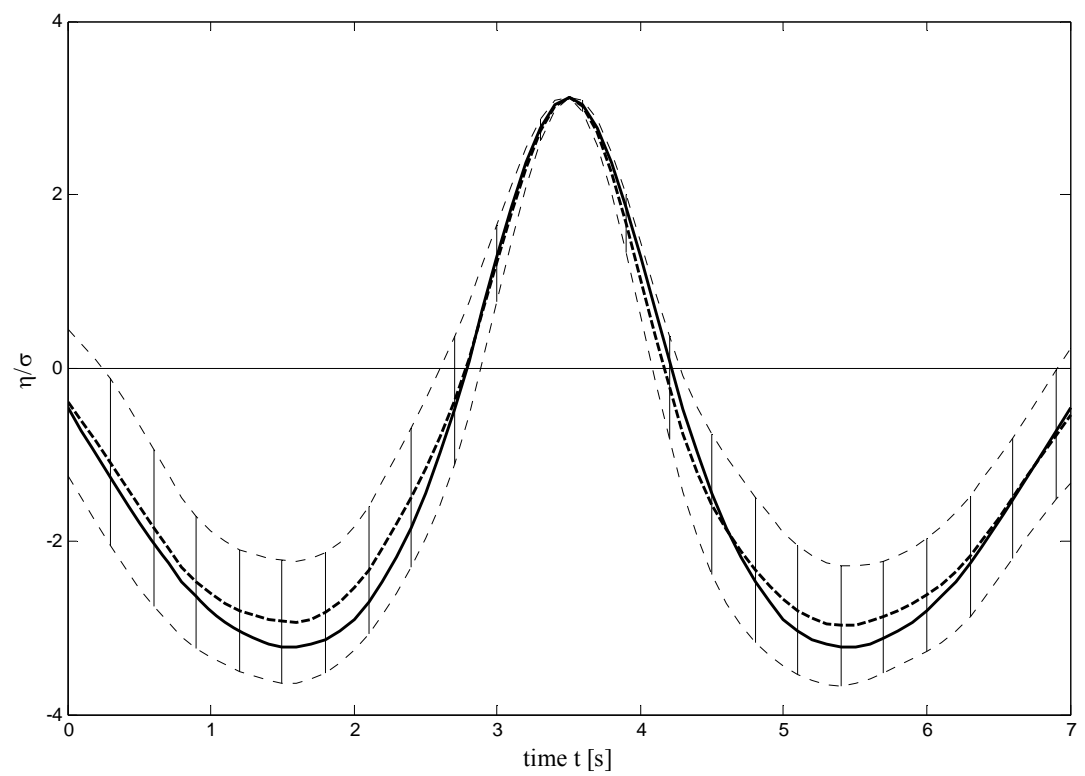

Fig. 11. Acqua Alta, Experiment 2: Observed shape of large waves (dashed line) given the crest amplitude $h$ in $[3.75 \sigma, 4.5 \sigma]$ and respective stability bands, and theoretical expected wave profile of Eq. (11) (solid line).

\subsection{Space extremes}

Consider a 2-D snapshot $\eta(x, y)$ of the wave surface at a given time $t$ over a given rectangular region $A$ of dimensions $l_{x}$ and $l_{y}$ as that shown in Fig. 12 from Experiment 3 at Acqua Alta. The excursion set $U_{\eta h}=\{(x, y) \in A: \eta(x, y)>h\}$ is the portion of $A$ over which $\eta$ is above the threshold $h$ (see Fig. 13). For this set, the Euler characteristic $E C$ is defined as the difference between the number of connected components and holes of the given set. If the threshold is low, then $E C$ counts the number of holes in the given set. If the threshold is high, then all the holes tend to disappear and the EC counts the number of connected components, or local maxima of the wave surface. For weakly nonlinear wave surfaces, the expected value of the $E C$ is given by (Adler, 1981; Worsley, 1995; Adler and Taylor, 2007, see also Fedele et al. 2011a; Fedele et al. 2011b; Fedele, 2012)

$<E C(\xi)>=E C_{a}\left(\xi_{1}\right)+E C_{b}\left(\xi_{1}\right)+\operatorname{Pr}\left\{Z>\xi_{1}\right\} \#$

where

$$
E C_{a}=\tilde{M}_{2} \xi_{1} \exp \left(-\xi_{1}^{2} / 2\right), E C_{b}=\tilde{M}_{1} \exp \left(-\xi_{1}^{2} / 2\right) . \#
$$

Here, $<\bullet>$ denotes expectation, $\xi=h / \sigma$ is a normalized threshold amplitude which satisfies $\xi=\xi_{1}+\mu\left(\xi_{1}\right)^{2} / 2$ and $Z$ is Gaussian with zero mean and unit variance. Further,

$\tilde{M}_{2}=\sqrt{2 \pi} \frac{l_{x} l_{y}}{\bar{L}_{x} \bar{L}_{y}} \sqrt{1-\alpha_{x y}^{2}}, \quad \tilde{M}_{1}=\frac{l_{x}}{\bar{L}_{x}}+\frac{l_{y}}{\bar{L}_{y}} . \#$

are the average number of 'waves' over the area $A$ and along the perimeter $P$, respectively, and $\bar{L}_{x}$ and $\bar{L}_{y}$ are the mean wavelengths along two orthogonal directions $x$ and $y$, respectively. Note that the wave parameters in (12)-(14) can be expressed solely in terms of the moments of the directional spectrum (see Appendix). The $E C_{b}$ term in (12) will be referred to as a boundary correction because it is important when the excursion set touches the boundary/perimeter of the region $A$ (Worsley 1995). 


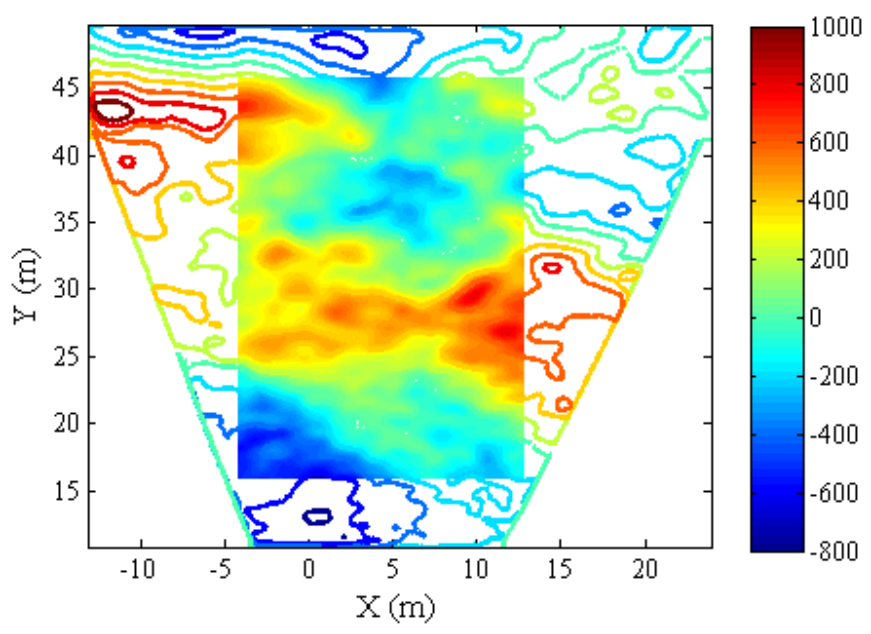

Fig. 12. Acqua Alta, Experiment 3: selected region $A$ of the wave surface elevation map (height in millimeters).

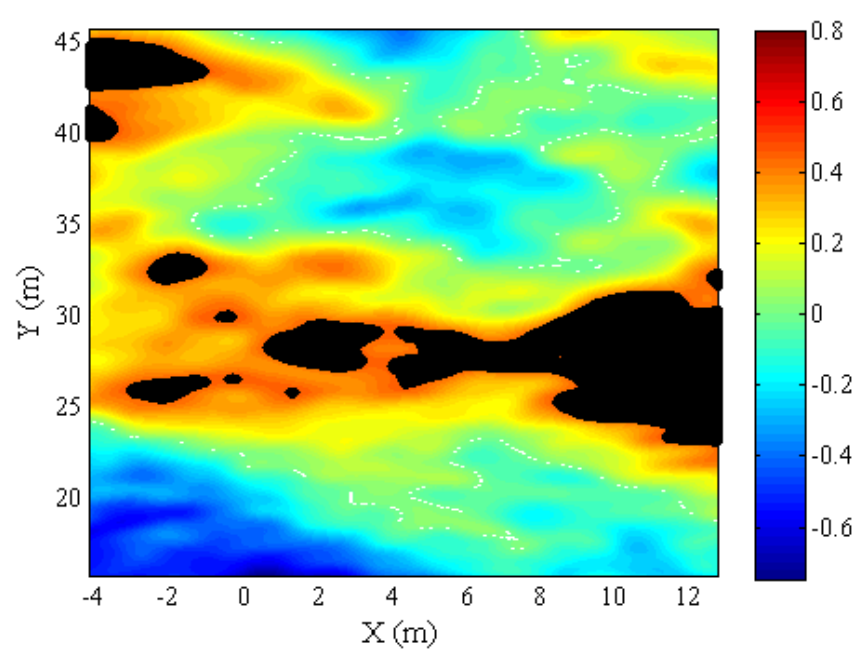

Fig. 13. Acqua Alta, Experiment 3: Excursion set (black region) of the normalized wave surface $\eta / \sigma$, that is the portion of domain $A$ over which $\eta>1.7 \sigma$. Note that, as the threshold increases the excursion set is the union of isolated regions delimiting the local maxima of the wave surface.

Adler (1981) and Adler and Taylor (2007) have shown that the probability that the global maximum of a random field $\eta$ exceeds a threshold $h$ is well approximated by the expected $E C$ of the excursion set $U_{\eta, h}$, provided the threshold is high. Indeed, as the threshold $h$ increases, the holes in the excursion set disappear until each of its connected components includes just one local maximum, and the $E C$ counts the number of local maxima. For very large thresholds, the $E C$ equals 1 if the global maximum exceeds the threshold $h$ and 0 if it is below. Thus, heuristically the $E C\left(U_{\eta, h}\right)$ of large excursion sets is a binary random variable with states 0 and 1 , from which follows that the probability of exceedance of the normalized global maximum $\xi_{\max }=\eta_{\max } / \sigma$ of $\eta$ over $A$ is given, for $\xi>>1$, by

$\operatorname{Pr}\left\{\xi_{\max }>\xi\right\}=\operatorname{Pr}\left\{E C\left(U_{\eta, h}\right)=1\right\}=<E C(\xi)>$.\#

Further, according to Gumbel (1958) the expected value of is given by

$$
<\xi_{\max }>=h_{N}+\frac{\mu}{2} h_{N}^{2}+\gamma_{e} \frac{1+\mu h_{N}}{h_{N}-\frac{\tilde{M}_{2}}{\tilde{M}_{2} h_{N}+\tilde{M}_{1}}}, \text { \# }
$$

where $h_{N}$ satisfies $\left(\tilde{M}_{2} h_{N}+\tilde{M}_{1}\right) \exp \left(-h_{N}^{2} / 2\right)=1$. If $\tilde{M}_{1}=0$, the Tayfun-Piterbarg model is recovered (Krogstad et al. 2004).

Fig. 14 reports the time variation of the estimated expected maximum $<\eta_{\max }>$ from Eq. (16) computed both with and without boundary corrections $\left(\widetilde{M}_{1} \neq 0\right.$ and $\widetilde{M}_{1}=0$ respectively). The two expectations slightly differ by $5-6 \%$. Clearly, these estimates are larger than the maximum height expected solely along the boundary of $A$. Note also that the observed maximum height over the entire area $A$ is bounded by the theoretical expected values from Eq. (16).

Further, we estimated the $E C$ for each of the reconstructed snapshots of the wave surface (9000 in total). The associated observed expected $E C$ value is reported in Fig. 15 together with the respective stability bands. Good agreement with their theoretical expectation (12) is found over small-to-moderate thresholds if the boundary corrections $E C_{b}$ are accounted for. Their effects tend to monotonically diminish at large thresholds as an indication that very large extremes most likely occur within the area $A$ and not on the boundary. This is consistent with the small differences noted in Fig. 14 between the expected values of the maximum height estimated with and without boundary corrections. Further, from Fig. 16 it is also clear that the asymptotic $E C$-based exceedance probability of the wave surface maximum (valid for $\eta>2.5 \sigma$ ) deviates from both the Rayleigh and Tayfun-Fedele models (see also Fedele et al., 2011a: Fedele et al., 2011b, Fedele, 2012). This provides evidence that the expected maximum surface height estimated over the imaged area is considerably larger than the expected maximum crest at a single point in space.

\subsection{Space-time extremes}

In the following we will investigate the extreme value statistics of the largest amplitude of the wave surface $\eta$ over a given area and time duration. To do so, consider the sequence $\left\{\xi_{1}, \xi_{2}, \ldots, \xi_{N}\right\}$ of the instantaneous normalized maximum $\xi_{\max }(t)=\eta_{\max }(t) / \sigma$ of $\eta$ observed over a region of the field of view of area $A$ at the generic time $t$. As a reference statistics, consider that of the expected maximum crest height $C_{\beta}(N)$ of $N$ waves whose parent statistical distribution follows the general law

$$
P(h>z)=z_{1}^{\beta} \exp \left(-z_{1}^{2} / 2\right), \beta \geq 1
$$

where the linear amplitude $z_{1}$ satisfies the Tayfun quadratic equation $z=z_{l}+\mu\left(z_{l}\right)^{2} / 2$ (Tayfun, 1986, see also Fedele, 2009) to account for second order nonlinearities. In this case, the expected maximum crest height depends upon the parameter and it is given, according to Gumbel (1958), by

$$
C_{\beta}(N)=h_{N}+\frac{\mu}{2} h_{N}^{2}+\gamma_{e} \frac{1+\mu h_{N}}{h_{N}-\beta / h_{N}},
$$

where $h_{N}$ satisfies $\left(h_{N}\right)^{\beta} \exp \left(-\left(h_{N}\right)^{2} / 2\right)=1 / N$. For $\beta=0$ the Tayfun model is recovered, as it should be. The 2-D and 3-D TayfunPiterbarg models (Krogstad et al., 2004) are obtained for $\beta$ equal to 1 and 2, respectively. The expected maximum $\xi_{\max }(N)$ over $N$ snapshots is computed according to the Gumbel statistics $C_{\beta}(N)$ of Eq. 18 and it is compared against the observed expected maximum wave elevation in Fig. 17 (data from Acqua Alta, 


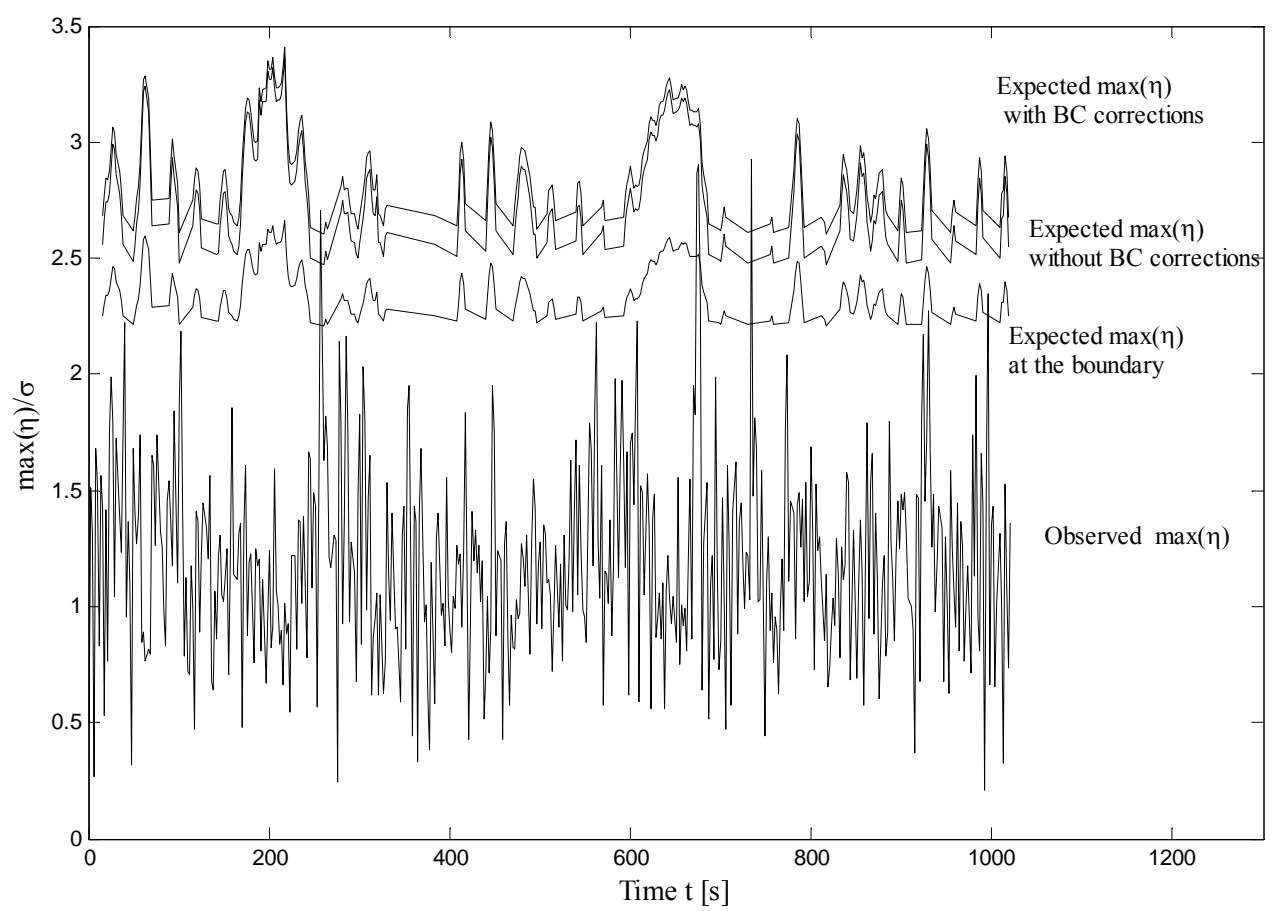

Fig. 14. Acqua Alta, Experiment 3: Time variation of the expected maximum wave surface over the large area $A$ of Fig. 12 computed with and without boundary corrections, the expected maximum along the boundary of $A$ and the observed maximum over the area from data.

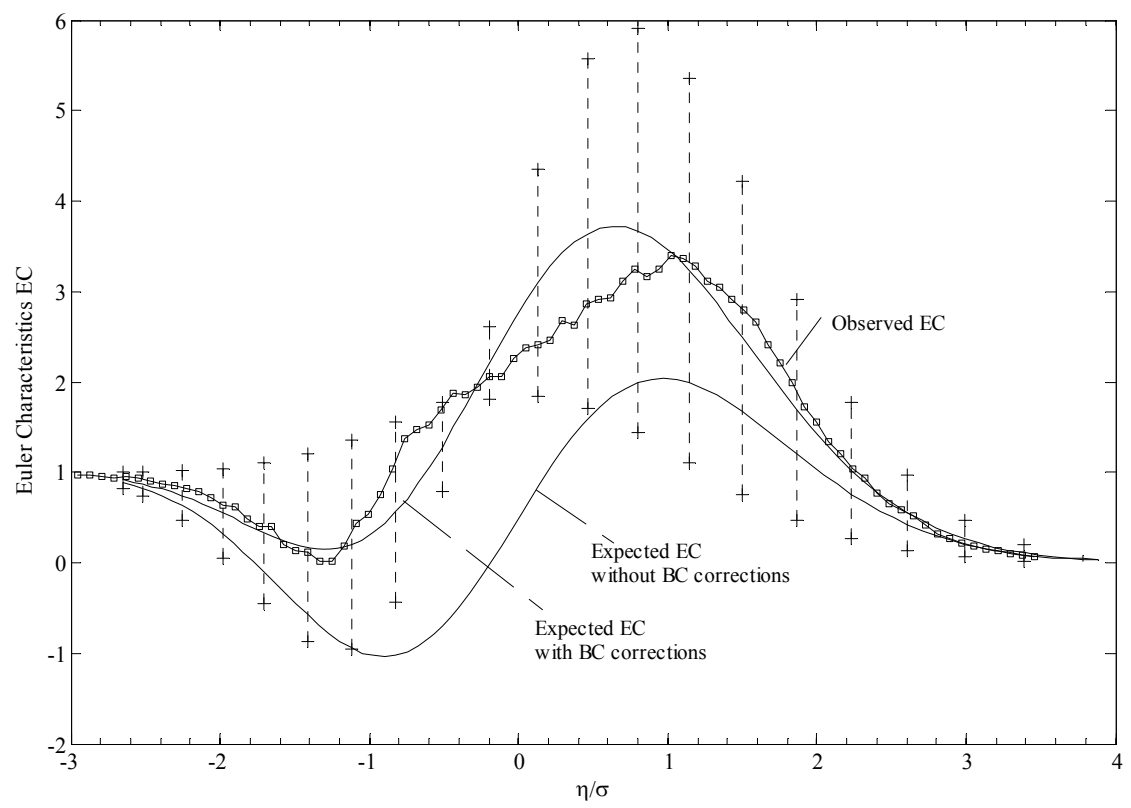

Fig. 15. Acqua Alta, Experiment 3: Observed Euler Characteristics and theoretical expected values over the area $A$ of Fig. 12.

Experiment 2). As the area increases, the observations deviate from the 1-D Tayfun model staying between the 2-D and 3-D Tayfun-Piterbarg statistics.

Note that the surface maximum estimates over the smallest area $\left(A=3 \times 10^{-3} \mathrm{~m}^{2}\right)$ are practically those observed at a given point, and hereafter referred to as $\xi_{\text {max,p. }}$. Indeed, in this case the mean wavelength $L_{m}$ is much larger than the length $A^{1 / 2}$ of the area's side. Clearly, as the area increases, so does the expected areal maximum $\xi_{\text {max }}$. For example, $\xi_{\text {max }} \approx 1.34 \xi_{\text {max }, \mathrm{p}}$ over the area $A \sim$ $534 \mathrm{~m}^{2}$. More rigorous stochastic models can be applied to describe space-time extremes and their expected values (Piterbarg, 1995; Adler and Taylor, 2007). Indeed, the wave surface can be modeled as a 3-D homogeneous Gaussian random field $\eta(x, y, t)$ over a space-time volume $\Omega$ of dimensions $l_{x}, l_{y}$ and $D$, respectively. According to Fedele (2012), in a Gaussian sea state of duration $D$ the expected maximum wave surface height $\xi_{\max }=\bar{\eta}_{\max } / H_{s}$ over the area $A=l_{x} l_{y}$ is given, according to Gumbel (1958), by

$$
\xi_{\max }=\zeta_{0}+\frac{\gamma_{e}}{16 \zeta_{0}-\frac{F^{\prime}\left(\zeta_{0}\right)}{F\left(\zeta_{0}\right)}}
$$

where $H_{s}$ is the significant wave height of the sea state, the prime denotes derivative and $\xi_{0}$ satisfies

$$
F(\zeta) \exp \left(-8 \zeta^{2}\right)=1
$$

with

$$
F(\zeta)=16 M_{3} \zeta^{2}+4 M_{2} \zeta+M_{1} .
$$




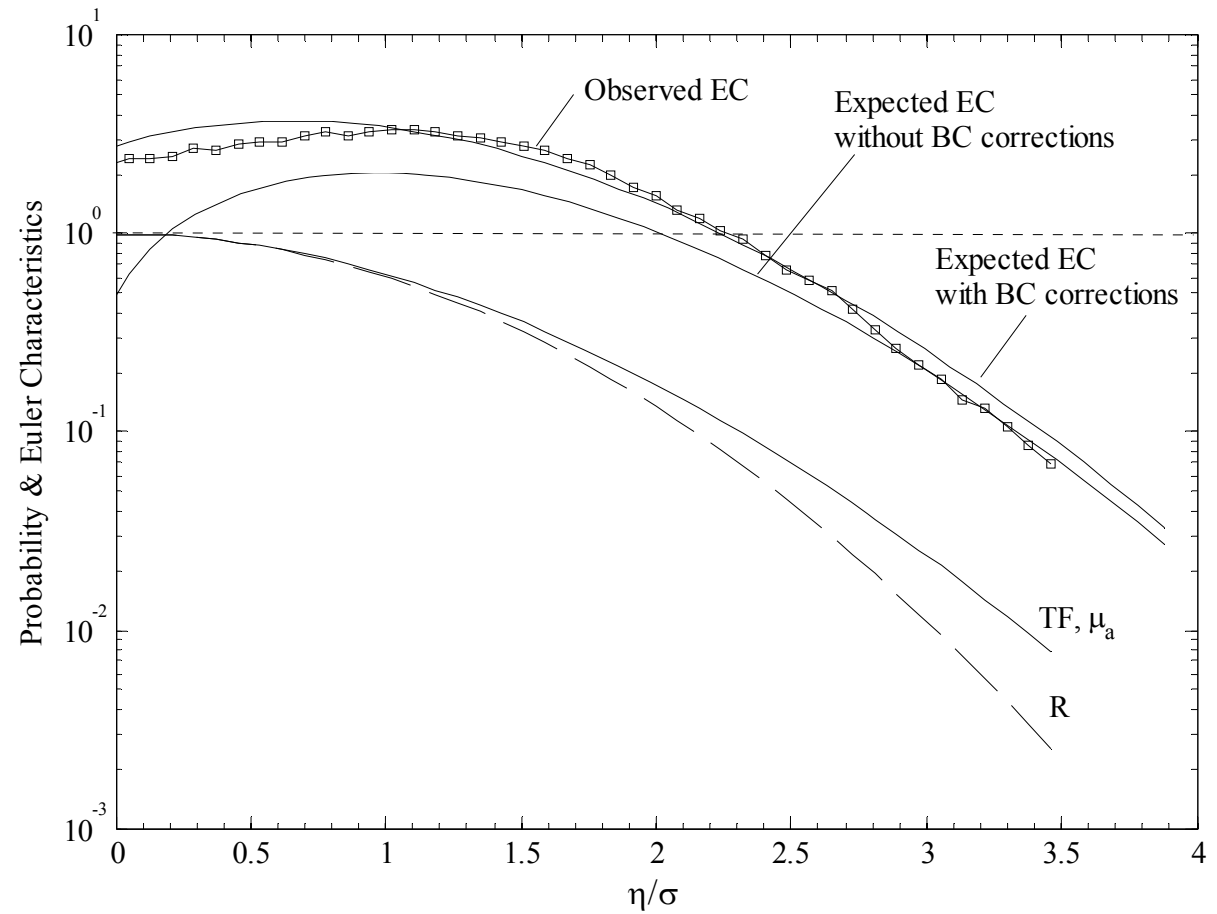

Fig. 16. Acqua Alta, Experiment 3: Observed Euler Characteristics and theoretical expected values compared to the $\mathrm{R}=\mathrm{Rayleigh}$ and $\mathrm{TF}=\mathrm{Tayfun}-\mathrm{Fedele}$ models, respectively.

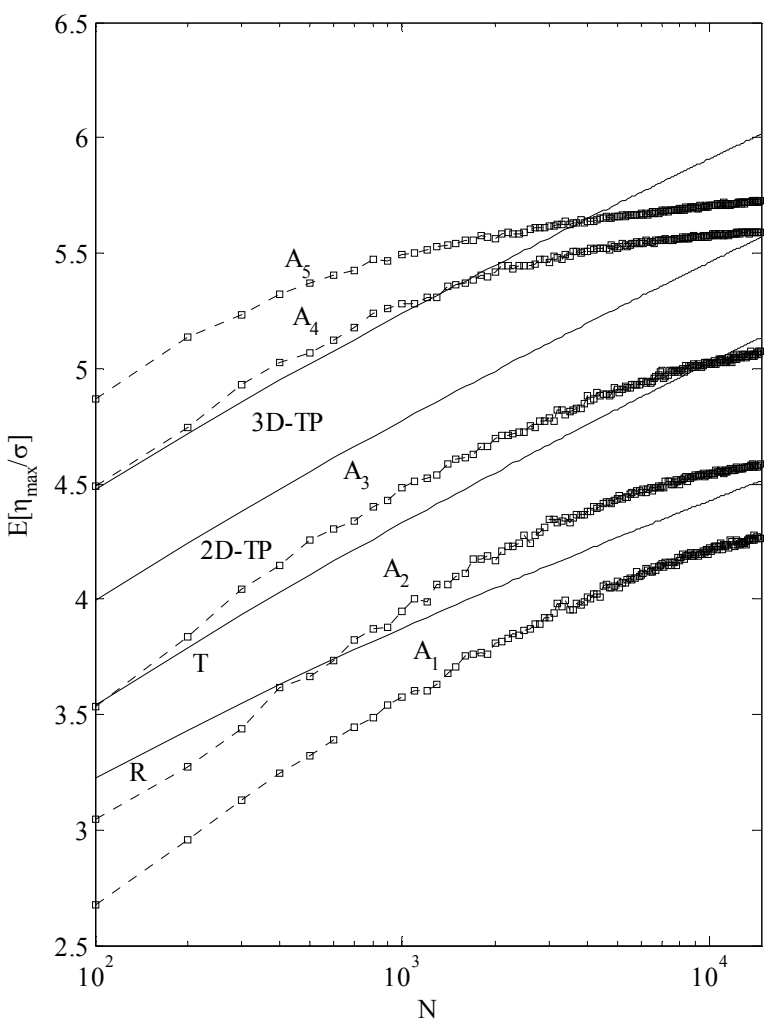

Fig. 17. Acqua Alta, Experiment 2: expected maximum of the surface height $\eta_{\max } / \sigma$ over the area $A$ as function of the number $N$ of snapshots against theoretical models $\mathrm{A}_{1}=3 \times 10^{-3}, \mathrm{~A}_{2}=1, \mathrm{~A}_{3}=10, \mathrm{~A}_{4}=100$, and $\mathrm{A}_{5}=534 \mathrm{~m}^{2}$. $\mathrm{R}=$ Rayleigh, $\mathrm{T}=$ Tayfun, $\mathrm{TP}=$ Tayfun-Piterbarg.

Here, $M_{3}, M_{2}$ and $M_{1}$ are the average number of 3-D, 2-D and 1$\mathrm{D}$ waves that can occur in the space-time volume spanned by the area $A$ during $D$. They all depend upon the directional wave spectrum and are given in appendix A. The expected maximum crest height $\xi_{\text {max,p }}$ at a given point follows from Eq. (19) setting
$M_{3}=M_{2}=0$. Thus, we can also estimate the ratio between the expected surface maximum $\xi_{\max }$ and that at a point, viz. $\xi_{\max , \mathrm{p}}$, as function of the area $A$. For example, in Fig. 18 we report the ratio $r=\xi_{\max } / \xi_{\text {max }, \mathrm{p}}$ observed from WASS measurements of the surface height (just one realization), which fairly agree with the theoretical estimates based on Eq. (19). In particular, $r \approx 1.26$ for $A=534 \mathrm{~m}^{2}$ in agreement with the predictions of Fig. 17 .

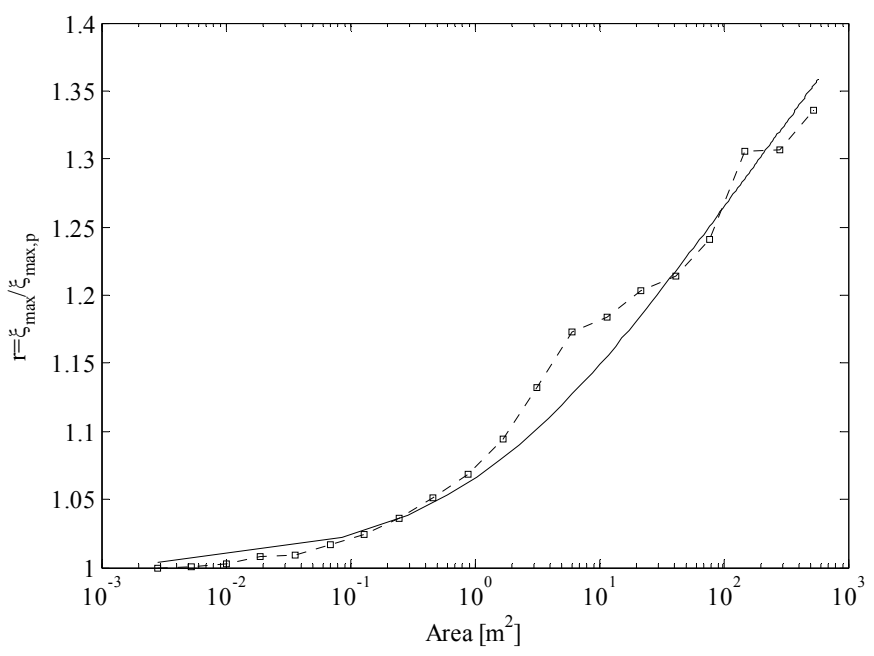

Fig. 18. Acqua Alta, Experiment 2: (solid line) theoretical predictions of the ratio $r=\xi_{\max } / \xi_{\text {max }, \mathrm{p}}$ between the expected maximum surface height $\xi_{\max }$ over an area and that expected at a point, viz. $\xi_{\text {max,p }}$ and (squares) observed $W A S S$ ratios from the measured sea state.

\section{Conclusions}

As a video observational technology, WASS is able to infer the space-time dynamics of oceanic states. To do so, stereo techniques based on the epipolar geometry and variational methods are exploited to obtain 3-D reconstructions of the sea surface map in time. By just elaborating time series of 
reconstructed wave surface displacements extracted at virtual point probes, we showed that the observed shape of large waves is predicted by stochastic models based on the theory of quasideterminism of Boccotti (2000). The estimated steepness of such large crests suggest that they do not violate the Stokes-Miche upper limit. Furthermore, a statistical analysis of the reconstructed spatial snapshots based on Adler's Euler Characteristics revealed that the maximum wave surface height over an area during a given duration (space-time extreme) is larger than that expected at a given point in space (time extreme). If the area is large enough compared to the mean wavelength, a space-time extreme most likely coincides with the crest of a focusing wave group that passes through the area. Clearly, the presented work demonstrates for the first time that the space-time wave statistics can be obtained from stereo video imaging and that existing stochastic theories of wave extremes agree with measurements.

\section{Acknowledgments}

The experiment at Acqua Alta was supported by Chevron (CASE-EJIP Joint Industry Project \#4545093). The Crimean experiment was supported The French Space Agency (CNES) and analysis is supported by the National Ocean Partnership Program, through the U.S. Office of Naval Research (Grant BAA 09-012). G. Gallego is supported by the Marie-Curie COFUND Programme of the EU and partially supported by the Ministerio de Ciencia e Innovación of the Spanish Government under project TEC2010-20412 (Enhanced 3DTV).

\section{Appendix A}

For space-time extremes, the coefficients in Eqs. (19)-(21) are given by (see, for example, Fedele, 2012)

$M_{3}=2 \pi \frac{D}{\bar{T}} \frac{I_{x} I_{y}}{\overline{L_{x}}} \alpha_{x y t}$

$M_{2}=\sqrt{2 \pi}\left(\frac{D I_{x}}{\bar{T} \overline{L_{x}}} \sqrt{1-\alpha_{x t}^{2}}+\frac{D I_{y}}{\bar{T} \overline{L_{y}}} \sqrt{1-\alpha_{y t}^{2}}+\frac{I_{x} I_{y}}{\overline{L_{x}} \frac{\bar{L}}{L_{y}}} \sqrt{1-\alpha_{x y}^{2}}\right)$,

$M_{1}=\frac{D}{\bar{T}}+\frac{I_{x}}{\bar{L}_{x}}+\frac{I_{y}}{\overline{L_{y}}}$.

which depend upon mean wave period $T$, mean wavelengths in $X$ and $Y$ directions, $\overline{L_{x}}$ and $\overline{L_{y}}$, viz.

$\bar{T}=2 \pi \sqrt{\frac{m_{000}}{m_{002}}}, \overline{L_{x}}=2 \pi \sqrt{\frac{m_{000}}{m_{200}}}, \overline{L_{x}}=2 \pi \sqrt{\frac{m_{000}}{m_{020}}}$

and

$\alpha_{x y t}=\sqrt{1-\alpha^{2}{ }_{x t}-\alpha^{2}{ }_{y t}-\alpha^{2}{ }_{x y}+2 \alpha_{x t} \alpha_{y t} \alpha_{x y}}$.

Here, $m_{i j k}$ are the moments of the directional spectrum $S(f, \theta)$ given by

$m_{i j k}=\iint k_{x}^{i} k_{y}^{j} f^{k} S(f, \theta) d f d \theta$

For space extremes $\left(D=0\right.$ and $\left.\alpha_{x t}=\alpha_{y t}=\alpha_{x y t}=0\right)$ from (A.1) the parameters $\tilde{M}_{1}$ and $\tilde{M}_{2}$ in Eq. (14) are recovered.

\section{References and notes}

Adler, R.J., 1981. The Geometry of Random Fields, John Wiley, 275 pp.

Adler, R.J., 2000. On excursion sets, tube formulae, and maxima of random fields. Annals of Applied Probability 10,1-74.

Adler, R.J., Taylor, J.E., 2007. Random fields and geometry, Springer Monographs in Mathematics, Springer, $454 \mathrm{pp}$.

Alvarez, L., Deriche, R., Sanchez, J., and Weickert, J., Dense disparity map estimation respecting image discontinuities : A pde and scale-space based approach. Journal of Visual Communication and Image Representation, vol. 13, pp. 3-21, 2002.

Banner, M. L., Jones, S. F., Trinder, J. C., 1989. Wavenumber spectra of short gravity waves. Journal of Fluid Mechanics 198, 321-344.

Baxevani, A., Richlik, I., 2004. Maxima for Gaussian seas. Ocean Engn., 33(7), 895-911.

Bechle, A.J., Wu, C.H., 2011. Virtual wave gauges based upon stereo imaging for measuring surface wave characteristics. Coastal Engineering 58(4), 305-316.

Benetazzo, A., 2006. Measurements of short water waves using stereo matched image sequences. Coastal Engineering 53, 1013-1032.

Benetazzo, A., Fedele, F., Gallego, G., Shih, P.C., Yezzi, A., 2012. Offshore stereo measurements of gravity waves. Coastal Engineering 64, 127 138.

Boccotti, P., 2000. Wave mechanics for ocean engineering. Elsevier Science, $496 \mathrm{pp}$.

Briggs, W. L., Henson, V. E., McCormick, S. F., A Multigrid Tutorial, 2nd Edition. SIAM, 2000.

Dankert, H., Horstmann, J., Lehner, S., Rosenthal, W.G., 2003. Detection of wave groups in SAR images and radar image sequences. IEEE Transactionson Geoscience and Remote Sensing 41, 1437-1446.

de Vries, S., Hill, D.F., de Schipper, M.A., Stive M. J. F., 2011. Remote sensing of surf zone waves using stereo imaging. Coastal Engineering 58(3), 239-250.

Fedele, F. 2005. Successive wave crests in Gaussian seas. Probabilistic Engineering Mechanics 20(4), 355-363.

Fedele, F. 2008. Rogue Waves in Oceanic Turbulence. Physica D 237(1417), 2127-2131.

Fedele F., 2009. On the statistics of oceanic waves. Inter. Journal of Reliability and Safety 3(1-3), 258-266.

Fedele, F., Gallego, G., Benetazzo, A., Yezzi, A., Sclavo, M., Bastianini, M., Cavaleri, L., 2011a. Euler Characteristics and Maxima of Oceanic Sea States. Journal Mathematics and Computers in Simulation 82(6):11021111 .

Fedele, F., Benetazzo, A., and Forristall, G.Z., 2011b. Space-time waves and spectra in the Northern Adriatic Sea via a Wave Acquisition Stereo System. In the Proc. of the 30th ASME Int. Conf. Offshore Mechanics and Arctic Eng., Rotterdam, The Netherlands, paper OMAE201149924.

Fedele, F., Tayfun, M.A., 2009. On nonlinear wave groups and crest statistics. J. Fluid Mech. 620, 221-239.

Fedele, F., 2012. Space-time extremes of short-crested storm seas. J. Phys. Oceanogr. 42, 1601-1615.

Forristall, G.Z. 2006. Maximum wave heights over an area and the air gap problem, OMAE2006-92022 paper, Proc. ASME 25th Inter. Conf. Off. Mech. Arc. Eng., Hamburg.

Forristall, G.Z., 2007. Wave crest heights and deck damage in Hurricanes Ivan, Katrina and Rita, Offshore Technology Conference Proceedings, OTC 18620, Houston.

Gallego, G., Yezzi, A., Fedele. F., Benetazzo, A. 2011. A Variational Stereo Algorithm for the 3-D reconstruction of ocean waves. IEEE Transations on Geoscience and Remote Sensing, 49(11), 4445-4457.

Gallego, G. Variational image processing algorithms for the stereoscopic space-time reconstruction of water waves. Ph.D. Thesis. Georgia Institute of Technology. May 2011.

Goring, D.G., Nikora, V.I., 2002. Despiking acoustic doppler velocimeter data. Journal of Hydraulic Engineering 128, 117-126.

Gumbel, E. J., 1958: Statistics of Extremes. New York: Columbia University Press, 1-373.

Hartley, R. and Sturm, P., "Triangulation", Computer Vision and Image Understanding, vol 68 (2), pp. 146-157, 1997.

Hashimoto, N., Nagai, T., Asai, T., 1994. Extension of the Maximum Entropy Principle Method for Directional Wave Spectrum Estimation. Proc 24th Int. Conf. Coastal Engineering (Kobe, ACSCE), pp 232-246.

Holland, K.T. and R.A. Holman. 1997. Video estimation of foreshore topography using trinocular stereo. Journal of Coastal Research 13(1): 81-87.

Holland, K.T., R.A. Holman, T.C. Lippmann, J. Stanley, and N. Plant. 1997. Practical use of video imagery in nearshore oceanographic field studies. IEEE Journal of Oceanic Engineering 22(1): 81-92. 
Holthuijsen, L.H., 1983. Observations of the directional distribution of ocean wave energy. J. Phys Oceanogr. 13, 816-827.

Jahne, B., 1993. Spatio-Temporal Image Processing - Theory and Scientific Applications, Springer-Verlag, 208 pp.

Johnson, D., 2004. DIWASP, a directional wave spectra toolbox for MATLAB ${ }^{2}:$ User Manual. Research Report WP-1601-DJ (V1.1), Centre for Water Research, University of Western Australia.

Kosnik, M.V., and Dulov, V.A., 2011. Extraction of short wind wave spectra from stereo images of the sea surface. Meas. Sci. Technol. 22, 1-10.

Krogstad, H.E., Liu, J., Socquet-Juglard H., Dysthe K.B. and K. Trulsen, 2004. Spatial Extreme Value Analysis of Nonlinear Simulations of Random Surface Waves, OMAE2004-51336 paper, Proc. ASME 23th Inter. Conf. Off. Mech. Arc. Eng., Vancouver.

Krogstad, H.E., Trulsen, K., 2010. Interpretations and observations of ocean wave spectra. Ocean Dynamics 60 (4), 973-991.

Lindgren, G., 1970. Some properties of a normal process near a local maximum. Ann. Math. Statist. 4(6),1870-1883.

Ma, Y., Soatto, S., Kosecka, J., Shankar Sastry, S., 2004. An invitation to 3D vision: from images to geometric models, Springer-Verlag, $526 \mathrm{pp}$.

Michell, J. H., 1893: On the highest waves in water. Philos. Mag., 5, 430437.

Newell, A.C., Zakharov, V.E., 2008. The role of the generalized Phillips' spectrum in wave turbulence. Phys Lett A 372 (23), 4230-4233.

Nobach, H., Honkanen, M., 2005. Two-dimensional Gaussian regression for sub-pixel displacement estimation in particle image velocimetry and particle position estimation in particle tracking velocimetry. Experiments in Fluids 38, 511-515.
Phillips, O.M., 1958. The equilibrium range in the spectrum of windgenerated waves, Journal of Fluid Mechanics 4(4), 426-434.

Phillips, O.M. 1977. The dynamics of the upper ocean, Cambridge University Press, $344 \mathrm{pp}$.

Piterbarg, V., 1995. Asymptotic Methods in the Theory of Gaussian Processes, AMS ser. Translations of Mathematical Monographs, Vol $148,205 \mathrm{pp}$

Rosenthal, W., Lehner, S., 2008. Rogue Waves: Results of the MaxWave Project. J. Offshore Mech. Arct. Eng. 130(2), 021006, doi: $10.1115 / 1.2918126$

Shemdin, O.H., Tran, H.M., Wu, S.C., 1988. Directional Mesurements of short ocean waves with stereography. J. Geophys. Res. 93,1389113901.

Tayfun, M.A., 1986. On narrow-band representation of ocean waves. Part I: Theory. J. Geophys. Res. 1(C6), 7743-7752.

Tayfun, M.A., Fedele, F., 2007. Wave-height distributions and nonlinear effects. Ocean Engineering 34(11-12), 1631-1649.

Trottenberg, U. Multigrid. Academic Press, 2000

Wanek, J.M., Wu, C.H., 2006. Automated trinocular stereo imaging system for three-dimensional surface wave measurements. Ocean Engineering 33(5-6), 723-747.

Worsley, K.J., 1995. Boundary corrections for the expected Euler characteristic of excursion sets of random fields, with an application to astrophysics. Adv. App. Prob. 27, 943-959.

Zakharov, V.E., 1999. Statistical theory of gravity and capillary waves on the surface of a finite-depth fluid. Eur. J. Mech. B Fluids 18(3), 327344. 